\title{
Free-riding and competition in network markets for digital goods
}

\author{
Andreas U. Schmidt \\ Fraunhofer Institute for Secure Information Technology SIT \\ Rheinstraße 75, 64295 Darmstadt, Germany \\ Email: andreas.schmidt@sit.fraunhofer.de
}

\begin{abstract}
We present a continuous, stochastic model - the first of its kind - for the monetary flow in multi-level markets allowing for the quantitative assessment of the incentive accruing to market participants through resales revenues. The main application of the model is for novel business models for the marketing of information goods, which purport to yield alternatives to technical copyright protection and DRM. We show analytically that within the scope of the model free-riding can be mitigated by employing multi-level marketing, and that multi-level markets are open markets for competing information goods.

C2008 IEEE. Personal use of this material is permitted. However, permission to reprint/republish this material for advertising or promotional purposes or for creating new collective works for resale or redistribution to servers or lists, or to reuse any copyrighted component of this work in other works must be obtained from the IEEE.
\end{abstract}

\section{INTRODUCTION}

Information goods are transferable and non-rival like public goods, and additionally are durable, i.e., show no wear out by usage or time [1], [2]. Like for a private good, however, original creation can be costly, whereas reproduction and redistribution are cheap. Thus, retail markets for information goods are plagued by free riding or 'piracy'. Hitherto, freeriders have been fought by technical methods for copyright protection and supporting legal regulation [3]-[5]. None of this has lead to sustainable success and economic, legal, and societal implications of rigid 'digital rights management' (DRM) raised a heated debate [6]-[8].

Alternative approaches to the of marketing virtual goods (a term coined by [9] and used for for information goods in intangible, digital form, and distributed via electronic networks) have gained importance. iTunes now offers media from major label EMI with superior quality and free of copy protection, in effect using the absence of DRM as a means of distinct quality discrimination [10]. Exponents of the computer and media industries issued statements raising doubts on the viability of DRM for media marketing [11], [12].

Most approaches still rest on conventional, centralised distribution structures. But some novel ideas have emerged drawing in fact from old ideas. Network marketing emulates the distribution system of free-riders, namely peer-to-peer systems [13]. It poses additional value proposition to buyers of the original (legal) version of the good by revenues or other rewards linked to resale. Important examples are Peer Impact [14], where buyers receive rebates on subsequent acquisitions for every resale, and the Potato System [15], [16], a genuine multi-level market for virtual goods. These ideas have not received the highest level of public attention, perhaps due to their similarity to illicit schemes, like pyramid and Ponzi schemes, or Peterand-Paul scams, and the like, cf. the detailed study [17].

Network and multi-level marketing in itself is underresearched apart from the mentioned European study. We have given first theoretical treatments of their market mechanisms, in particular for information goods, in [18], [19], including a raw model for competition in [19]. These preceding studies concentrated on the issue of fairness of network marketing, and economic characteristics of it in the case of information goods, respectively. Here, we present a generic model for single and multiple rewarding levels in a network market, including unlimited payments from the down-line. Moreover, we exhibit a detailed framework to describe competition in network markets, based on a micro-model for externalities of buyers and their decision-making. The aim is to answer the fundamental question: Can the original version of an information good prevail against free-riders, when distributed using an appropriate network market mechanism?

Section [II presents the model for the macroscopic mechanism of network markets of [18] and adds a Markov model for payments from the down-line, i.e., genuine multi-level rewards. In both cases we collect the most important features and provide an economic discussion in II-C. In Section III, a framework to describe the competition of information goods in network markets is systematically introduced. It is based on the various endogenous and exogenous factors influencing agent's buying decisions. Special experiments are carried out in Section IV, where the performance of competing goods is evaluated in terms of market shares, turnovers, and revenues accruing to a collector, e.g., the good's originator. The potential of network marketing to mitigate free-riding and a genuine case of competition are evaluated. Section $\mathrm{V}$ provides a summary discussion and directions for further work.

\section{Models for Network ANd Multi-level Markets}

In this section we first briefly recapitulate the model for the monetary flow in network markets developed in [18], [19], which is a core ingredient to the model for competition in the next section. Second, we consider genuine multi-level markets in which upstream payments to resellers come from many, or all, subsequent buyer generations. 


\section{A. Generic Network Markets}

In generic network markets goods are super-distributed from buyer, which then acts as a reseller, to buyer. An incentive to buy a good accrues to buyers/resellers by the resale revenues they can achieve. In a supply monopoly and a continuous setting this incentive is determined by the monetary flux model of [18], which we now recapitulate. Consider a market evolving in finite time $0 \leq t \leq \infty$ from size 0 to a finite size $n_{\infty}=n(\infty)$, and an agent, say $a$, entering at time $t$, i.e., buying the good from some other agent for a price determined by the price schedule $\pi(t)$. The influx of agents at any later time is $\dot{n}\left(t^{\prime}\right)=\mathrm{d} n\left(t^{\prime}\right) / \mathrm{d} t^{\prime}$, and at this time $a$ competes with $n\left(t^{\prime}\right)$ others for the newcomers as buyers. Thus the expected differential resale revenue of $a$ at a later time $t^{\prime}$ becomes

$$
\frac{\pi\left(t^{\prime}\right)}{n\left(t^{\prime}\right)} \dot{n}\left(t^{\prime}\right) \mathrm{d} t^{\prime}
$$

The full expectation of resale revenue for $a$ is

$$
v_{r}(t)=\int_{t}^{\infty} \frac{\gamma\left(t^{\prime}\right) \pi\left(t^{\prime}\right)-\tau}{n\left(t^{\prime}\right)} \dot{n}\left(t^{\prime}\right) \mathrm{d} t^{\prime},
$$

where the commission factor $0 \leq \gamma \leq 1$ accounts for the taxation of the resale price by a principal we call the collector, e.g., the original creator of the good or an intermediary collecting agency, who obtains $(1-\gamma(t)) \pi(t)$ from a resale at time $t$. The constant $\tau \geq 0$ subsumes the transaction cost an agent incurs in processing a single resale, and is neglected in the competition model below, as we concentrate on information goods. Transaction costs incurred by buyers are absorbed in the time-dependent price $\pi$. Reparametrisation by the saturation $s=n / n_{\infty}, s \in[0,1]$, yields (by integrating over the auxiliary coordinate $\sigma$ )

$$
v_{r}(s)=\int_{s}^{1} \frac{\gamma(\sigma) \pi(\sigma)-\tau}{\sigma} \mathrm{d} \sigma .
$$

In choosing $s$ instead of real time as dynamic coordinate it is assumed that no participants leave the market and thus $s$ is monotonic increasing in time. Furthermore we assume that the market is finite and saturation $s=1$ is reached in finite time, i.e., the transition from time to $s$ is nonsingular and invertible. The setting is scale-free in the sense that its dynamics does not depend on the absolute market size.

The basic properties of the monetary incentive $v_{i}(s) \stackrel{\text { def }}{=}$ $v_{r}(s)-\pi(s)$, obtained from the resale revenues (3), and the operator $K: \pi \longmapsto v_{i}$ are the following. As $K$ describes a monetary flux between market agents, as well as from agents to collectors and into transaction costs, one would expect it to satisfy a 'hydrodynamic' balancing condition. If $\pi$ is bounded, then the incentive satisfies [18, Proposition 1]

$$
\int_{0}^{1} v_{i}(\sigma) \mathrm{d} \sigma=\int_{0}^{1}(\gamma(\sigma)-1) \pi(\sigma) \mathrm{d} \sigma-\tau .
$$

For $\gamma=1$, and $\tau=0$ this law takes the form of a gametheoretical zero-sum condition, expressing that wins and losses in incentive compensate exactly.

$$
\int_{0}^{1} v_{i}(s) \mathrm{d} s=0
$$

For regular enough $\pi$, the inverse of $K$ is easily obtained as a solution of the inhomogeneous equation $K \pi=v_{i}$. We denote the derivatives of $\pi, v_{i}$, with $\dot{\pi}, \dot{v}_{i}$, respectively, and under certain regularity conditions we obtain the inverse $\check{K}[18$, Proposition 2]

$$
\check{K} v_{i}(s) \stackrel{\text { def }}{=}-E_{\gamma}(s)^{-1} \int_{0}^{s} \dot{v}_{i}(\sigma) E_{\gamma}(\sigma) \mathrm{d} \sigma+\frac{\tau}{\gamma(s)},
$$

with the abbreviation $E_{\gamma}(s) \stackrel{\text { def }}{=} \exp \left(\int_{0}^{s} \gamma(\sigma) / \sigma \mathrm{d} \sigma\right)$. This formula enables the use of $\pi$ to determine the incentive by dynamic forward pricing and therefore as a lever for competitive discrimination.

Although nothing in principle prevents a monetary flow from earlier buyers to later ones by negative prices, the conventional case is that of positive prices, characterised in [18, Proposition 3]. The result has a rather direct interpretation in that the monetary flow is always directed backwards if and only if the expected incentive at a certain time is smaller than the average expected incentive before that time.

A continuous model is an idealisation of a real market where buyers enter one by one, i.e., the market size evolves in discrete steps. This entails artifacts, most notably a logarithmic singularity for $v_{i}(s)$ as $s \searrow 0$ when $\pi(0)>0$. The discrepancy between the $v_{i}(s)$ and the discrete incentive $\bar{v}_{i}\left(s \cdot n_{\infty}\right)$ at the corresponding point is, for bounded, non-negative $\pi, \gamma=1$, $\tau=0$,

$$
\begin{aligned}
& \left|v_{i}(s)-\bar{v}_{i}\left(s n_{\infty}\right)\right| \leq \\
& \quad \frac{\pi_{\max }}{2}\left[\frac{1+s}{s n_{\infty}}+\frac{1}{6} \frac{1+s^{2}}{\left(s n_{\infty}\right)^{2}}+O\left(\frac{1+s^{4}}{\left(s n_{\infty}\right)^{4}}\right)\right],
\end{aligned}
$$

with $\pi_{\max } \stackrel{\text { def }}{=} \max _{s \in[0,1]} \pi(s)$, and in which each term is strictly dominated by the previous one, see [18, Proposition 4]. This error behaviour is rather benign in that it decays with the inverse of the market size at any finite saturation $s>0$. For fixed $k=s n_{\infty}$ on the other hand, a constant error bounded by $c_{k} \pi_{\max }$ for some $c_{k}>0$, will always remain.

\section{B. Multi-Level Markets}

The 'multi' in multi-level often refers to the fact that in many network markets, many subsequent levels, or generations, of buyers contribute to an agent's resales revenues, or even all of them. For instance, the Potato system [15], [16] allows for down-line payments from up to three subsequent buyer levels. Though we argue in [18, Section 3.1] that such may be undesirable from a policy viewpoint, we now complete our theory to account for multiple rewarding levels. The following derivation assumes the absence of upward discrimination, i.e., buyers pay the price according to the schedule $\pi(t)$, regardless of their, respectively their vendor's, level in the tree of buyers. Otherwise, buyers would be tempted to optimise their level, a market distortion we do not consider.

For agent $a$, the expected cumulated number of level 1, i.e., direct buyers at a later time $\tilde{t}>t$ is

$$
n_{1}(t, \tilde{t})=\int_{t}^{\tilde{t}} \frac{\dot{n}\left(t^{\prime}\right)}{n\left(t^{\prime}\right)} \mathrm{d} t^{\prime}=\int_{n(t)}^{n(\tilde{t})} \frac{\mathrm{d} n}{n}=\ln \frac{n(\tilde{t})}{n(t)} .
$$


According to the assumption above, the process generating subsequent buyer levels $>1$ is Markovian, entailing the general expression for $k \geq 1$,

$$
n_{k}(t, \tilde{t})=\int_{t}^{\tilde{t}} n_{k-1}(t, \tilde{t}) \frac{\dot{n}\left(t^{\prime}\right)}{n\left(t^{\prime}\right)} \mathrm{d} t^{\prime}=\frac{1}{k !}\left(\ln \frac{n(\tilde{t})}{n(t)}\right)^{k} .
$$

Let $0 \leq \gamma_{k} \leq 1$ be the commission factor for level- $k$ resales, $\gamma_{0}=\gamma$, and set $n_{0}=1$. For $N$ levels of reward beyond direct resales, the expected resales revenue can then be expressed, extending (2) and neglecting transaction costs, as

$$
v_{r}^{(N)}(t)=\sum_{k=0}^{N} \int_{n(t)}^{n_{\infty}} n_{k}\left(t, t^{\prime}\right) \frac{\gamma_{k}\left(t^{\prime}\right) \pi\left(t^{\prime}\right)}{n\left(t^{\prime}\right)} \mathrm{d} n\left(t^{\prime}\right) .
$$

Inserting (9) and reparametrisation by $s$ obtains

$$
v_{r}^{(N)}(s)=\int_{s}^{1} \frac{\pi(\sigma)}{\sigma} \sum_{k=0}^{N} \frac{\gamma_{k}(\sigma)}{k !}\left(\ln \frac{\sigma}{s}\right)^{k} \mathrm{~d} \sigma .
$$

The commission factors are taken as independent parameters. Thus they must satisfy

$$
1-\bar{\gamma}(s) \stackrel{\text { def }}{=} \sum_{k=0}^{N} \gamma_{k}(s) \leq 1 \quad \forall s
$$

since distributed rewards cannot exceed $\pi(s)$. The cumulative commission $\bar{\gamma}$ determines the remaining share of $\pi$ that can accrue to a collecting agency or the good's originator.

The case of infinite rewarding levels is often realised in network marketing organisations for physical goods. To treat the limit $N \rightarrow \infty$ note that the limit function

$$
\Gamma(s, z) \stackrel{\text { def }}{=} \sum_{k=0}^{\infty} \frac{\gamma_{k}(s \exp z)}{k !} z^{k}
$$

is defined on $[0,-\ln s]$, and measurable there, as a consequence of Lebesgue's theorem on monotonic convergence, since $0 \leq \gamma_{k} \leq 1, \forall k$. The function $\Gamma$ is positive and $\Gamma(s, 0)=\gamma_{0}(s)$. Under mild conditions on the decay of $\gamma_{k}$, for instance if $\gamma_{k}=$ const, $\Gamma$ is monotonic in $z$. We obtain

$$
v_{r}^{(\infty)}(s)=\int_{s}^{1} \frac{\pi(\sigma)}{\sigma} \Gamma(s, \ln (\sigma / s)) \mathrm{d} \sigma .
$$

The case $\gamma_{k}=q \cdot q^{k}$ with $q<1$ is instructive. That is, taxation by commissions initiates with a share $1-q$ and increases geometrically for down-line payments from higher levels. Then $\Gamma(z)=q \exp (q z)$ is independent of $s$ and

$$
v_{r}^{(\infty)}(s)=\frac{q}{s^{q}} \int_{s}^{1} \frac{\pi(\sigma)}{\sigma^{1-q}} \mathrm{~d} \sigma .
$$

\section{Economic properties}

Salient features of the model are the following [18], [19].
1) Illicit schemes: Multi-level marketing carries negative connotations and is illegal in special forms known as pyramid selling, snowball systems, chain-letters, etc., under many jurisdictions. [17, Vol. II] present criteria to distinguish between legitimate multi-level marketing and such practises that are to be considered illicit. The present model yields some arguments in favour of multi-level markets (extensively presented in [18], [19]. i) A buyer acquires not only a void right to resale, but with it a good of value. Potential losses an agent entering at a late stage will incur are charged up against this value. ii) Inventory loading, i.e., the obligation to keep a large, non-returnable stock, is irrelevant for information goods. iii) Marginal costs for replication and redistribution are mostly much smaller than resale prices and thus transaction costs are largely insignificant. iv) The main novel feature of the model presented above is that it enables a fair market mechanism design. As realistic information on achievable rewards is a crucial criterion for a scheme's legitimacy, the possibility to determine and publish price and expected incentive schedules becomes essential.

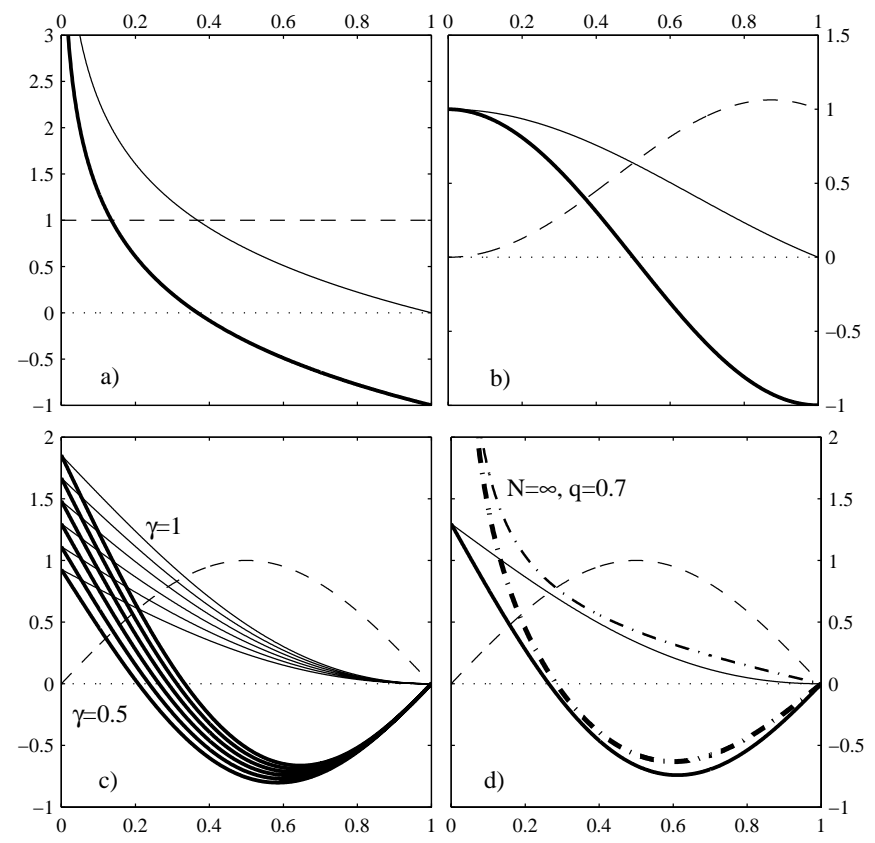

Fig. 1. Examples for prices $\pi$ (dashed), expected resale revenues $v_{r}$ (thin solid), and incentives $v_{i}$ (thick solid). For a)—c) $\gamma=1$. a) $\pi=1$, $v_{r}=-\ln s, v_{i}=-\ln s-1$. b) $\pi(s)=\sin (\pi s) /(\pi s)-\cos (\pi s)$, $v_{r}=\sin (\pi s) /(\pi s), v_{i}=\cos (\pi s)$. c) $\pi(s)=\sin (\pi s), v_{r}=\operatorname{Si}(\pi)-$ $\mathrm{Si}(\pi s), v_{i}=\mathrm{Si}(\pi)-\mathrm{Si}(\pi s)-\sin (\pi s)$, where $\mathrm{Si}$ is the integral sine function. Commission factor $\gamma$ varies from 1 to 0.5 in steps of size 0.1 . d) Comparison of infinite multi-level rewards (dash-dots) with level-0 rewards only at commision factor $\gamma=\gamma_{0}=q=0.7$.

2) Dynamic forward pricing: The principal of a good can, via the inversion formula (6), dynamically adapt the incentive during the evolution of the market (if $\pi$ is exogenous and controlled, e.g., by him). This possibility has not been considered for multi-level markets for information goods. Figure 1 shows basic price schedules and resulting incentives. Constant prices (a) are associated with favouritism of early 
adopters, increasingly penalising later market entrants. An early subscriber discount is shown in b), a schedule often used to counteract a slow startup. For virtual goods, such an initial invitation to enter can be important in the presence of freeriders, when early buyers cannot be sure about their potential resale revenues which depend logarithmically on the market size, as $v_{i}\left(k=s n_{\infty}\right)$ scales as $\ln n_{\infty}$. However, the particular price schedule (b) strongly disfavours late-comers.

The price schedule in (c) improves on (b) by letting the price vanish when the market reaches saturation, combining and early subscriber discount with a rebate for late-comers. This can help avoid slow startup and at spur the market at late times, when it tends to loose dynamics. Assuming that endogenous market growth is strongest at medium saturation and thus demand is high, it makes sense to let $\pi$ have its maximum there. (d) also shows the rather limited effect that a collector has on the incentive. Note that the logarithmic singularity is avoided if $\pi(0)=0$.

A proper determination of the incentive schedule can only be realised if the current market size $n(t)$ is known, as is the case in centralised systems like Potato. The total size $n_{\infty}$ however, remains a priori unknown, and can at best be estimated from past statistics. The price schedules in c) and d) suggest a pragmatic approach, namely to enforce closure of the market by letting $\pi$ approach 0 at a given $n_{\infty}$. Though this strategy runs counter to the aim of maximising collectors' revenues the effect on the turnover is limited if the price becomes small enough, e.g., by a rebate, at late times. A real-world instance for such price schedules is provided for scholarly information, as some academic publishers are distributing classic titles for free. Also, many legal codifications of intellectual property rights foresee a forfeiture after a certain period.

For mechanism design it is desirable to decouple the agents' decisions from the price and bind it to the incentive. A condition is true information about expected incentives, i.e., market transparency. Mixed forms of dynamic pricing can be envisaged, e.g., positive correlation of $\pi$ with the buying frequency, combined with a frequency or price threshold below which the price is set to zero and the market closed.

3) Scale-Freeness: The incentive $v_{i}$ is, for bounded $\pi$, independent of $n_{\infty}$. This scale-freeness of the model means that no effects can be modelled which depend on the absolute market size. In particular, aggregate network effects depending on $n_{\infty}$ are assumed to be negligible for the competition of goods, which is justifiable, e.g., if they affect each good to the same extent. The first buyer of the good, the root of the market, can expect a revenue growing as $O\left(\ln n_{\infty}\right)$ with the market size, while the collector imposing a commission can expect $O\left(\gamma n_{\infty}\right)$ if $\gamma$ is constant. This is in essence a consequence of the fact that a pure multi-level market cannot easily be monopolised by a single reseller, or a group of them. It explains why commission models are a standard practise in network marketing.

4) Network effects: It can sensibly be assumed that network and nulti-level markets are influenced by network effects, i.e., the benefit that accrues to a user of a good or a service because he or she is one of the many who use it. Simple functional forms of network effects for special types of networks, e.g., telecommunication networks, such as Sarnoff's, Metcalfe's, and Reed's law, are often heuristically used to explain the growth dynamics of networks. The most prominent phenomena traced back to network effects are a "slow startup", the existence of a "critical mass" [20], and strong (supraexponential) growth after it is exceeded. Models for network externalities and their economic impact are numerous and detailed, see, e.g., [21], [22], while global models, such as [23] for their possible functional forms, are scarce.

In network markets, the reward is an explicit network utility, but others cannot be excluded. Recent studies like [24] show in particular that consumer's choices are strongly influenced by information on choices of their peers, expressed for instance in the form of popularity rankings. Such perceived network utility must be accounted for in models of buyer behaviour. Moreover, the relative share of one good at a certain stage contributes to its visibility and in turn to the probability of matchmaking for its resale. This entails a positive network effect in any realistic model for the topology of the market.

5) Market (In)homogeneity: Multiplier effects are a prominent example for details which undermine one crucial assumption underlying the model presented, namely homogeneity of the market. Uniform agents in a structureless market are a good approximation if the number of potential participants is large and consists of a rather homogeneous group of individuals, for instance with special preferences. However, if the market is biased in the sense that there is a group of agents with higher trading capacities, the assumption breaks down. Large music labels running direct sale web sites are an example. Furthermore, inhomogeneities amplified by multiplier effects carry the imminent danger that the market can be cannibalised at an early stage by an agent with overwhelmingly high communication capacity, e.g., a popular web site, who could then obtain a practical monopoly. It is implied in [25] that monopoly creation could be a rather natural effect in Ecommerce. While the originator of the good is not too affected by this phenomenon if a commission model is used, other buyers' incentives are always negatively affected.

6) Multiple rewarding levels: Payments from the downline often occur in multi-level marketing of physical goods, but also in pure pyramid schemes. They yield rewards which do not arise from sales of the good and seemingly add an aleatory element. The main argument to their support is that competition between resellers and their direct buyers should be attenuated, since otherwise the subjective incentive to resell might be low. The study [17, Vol. II, p. 236] does not categorically condemn multi-level marketing, arguing that undesired effects to consumers can be effectively mitigated by appropriate mechanism design. Our analysis in Section IIB corroborates this view. Payments from the down-line yield an additional reward to all agents, which in our model is taken from the collector. The limit case of infinite rewarding levels, in the rather natural case of geometric commissions, shows explicitly how competition is alleviated by a factor $\sigma^{q}$, 
see (15). In the example of Figure 1 d), early adopters are the main beneficiaries of down-line payments, while agents in the last third of the market evolution still gain significantly from them.

\section{COMPETition MODEL}

This section specifies a mesoscopic model for the stochastic dynamics of two competing information goods. The macroscopic mechanism of network markets described above is used here as an externality in a generic micro-model for agents' buying decisions. We first specify the dynamic framework and then introduce the external utilities affecting buyers in a systematic way, differentiating between endogenous and exogenous factors.

\section{A. General Setting}

The situation we want to describe is perfect demand competition and a duopoly of suppliers. A duopoly of substitute goods $A$ and $B$ is described by the market shares

$$
s^{\bullet}(s), \bullet=A \text { or } B, \quad s^{A}+s^{B}=s .
$$

Potential buyers, called agents, enter the market at a constant rate and decide to buy either $A$ or $B$. The dynamic evolutions of $s^{\bullet}$ are thus determined by probabilities $\rho^{\bullet}(s)$ for the latter decision, i.e.,

$$
\frac{\mathrm{d} s^{\bullet}(s)}{\mathrm{d} s}=\rho^{\bullet}(s), \quad \rho^{A}(s)+\rho^{B}(s)=1 .
$$

The decision probability for $A$ is determined by spatial distributions of the individual von Neumann-Morgenstern utilities $u$ of $A$ and $B$ among the agent population as follows

$$
\rho^{A}=\operatorname{Pr}_{\mu^{A}, \mu^{B}}\left(u^{A}>u^{B}\right)=\int_{0}^{\infty} \mathrm{d} \mu^{A}\left(u^{A}\right) \int_{0}^{u^{A}} \mathrm{~d} \mu^{B}\left(u^{B}\right),
$$

and likewise for $B$, where $\mu^{\bullet}$ is a probability density function (PDF). This definition automatically satisfies (17).

We assume that agents act pursuant to a common decisionmaking mechanism. Accordingly, the random variables $u^{A}$ and $u^{B}$ are distributed with a common probability distribution. This distribution is determined by the PDF

$$
\mathrm{d} \mu \mu^{\bullet}\left(u^{\bullet}\right)=\mu\left(\left\{u_{\text {exogenous }}^{\bullet}(s)\right\},\left\{u_{\text {endogenous }}^{\bullet}(s)\right\} ; u^{\bullet}\right) \mathrm{d} u^{\bullet},
$$

which depends on a finite number of externalities which are either endogenous or exogenous factors with respect to the market at a given saturation. We generally assume that individual utilities in the absence of externalities are positive, that is

$$
\operatorname{supp}(\mu(\{\ldots, 0, \ldots\} ; u)) \subset[0, \infty) .
$$

Furthermore, $\mu$ shall satisfy the principle of stochastic dominance, separately in each externality, i.e.,

$$
\mathcal{M}\left(\left\{u_{i}\right\} ; u\right) \geq \mathcal{M}\left(\left\{v_{i}\right\} ; u\right) \quad \text { if } v_{i} \geq u_{i} \forall i,
$$

where $\mathcal{M}\left(\left\{u_{i}\right\} ; u\right)=\int_{0}^{u} \mathrm{~d} \mu\left(\left\{u_{i}\right\} ; u^{\prime}\right)$ is the cumulative density function $(\mathrm{CDF})$ of $\mu$. By stochastic dominance, $\rho^{\bullet}$ is monotonously increasing, separately in each externality.

\section{B. Model Specification}

In this general setting, a concrete model is determined by specifying $\left\{u_{\text {exogenous }}^{\bullet}\right\},\left\{u_{\text {endogenous }}^{\bullet}\right\}$, and $\mu$, which we now do in that order.

Exogenous factors: We introduce two exogenous variables $p^{\bullet}(s)$, called popularity, and $\pi^{\bullet}(s)$, called price. Together they constitute the single exogenous externality $p^{\bullet}(s)-\pi^{\bullet}(s)$. The two variables $p^{\bullet}$ and $\pi^{\bullet}$ contribute in different ways to the endogenous factors.

Endogenous factors: The first endogenous factor is an expected reward for buying either of the goods. It is calculated by a rule of bounded rationality using as basic information the current decision probabilities $\rho^{\bullet}(s)$, and an estimate $v_{r}^{\bullet}(s)$ for the reward for buying good $\bullet$ in a monopoly situation. The expected reward is then given by

$$
u_{r}^{\bullet}(s) \stackrel{\text { def }}{=} v_{r}^{\bullet}(s) \cdot \rho \cdot(s) .
$$

The monopoly reward is given by formula $(3)$, recast for both goods as

$$
v_{r}^{\bullet}(s) \stackrel{\text { def }}{=} \int_{s}^{1} \frac{\gamma^{\bullet} \pi^{\bullet}(\sigma)}{\sigma} \mathrm{d} \sigma,
$$

neglecting transaction costs, and with a constant commission factor $\gamma^{\bullet} \in[0,1]$. The second endogenous factor is a genuine, positive network externality, see Section II-C.4 dependent on a common, adjustable parameter $\varepsilon \geq 0$,

$$
u_{m}^{\bullet} \stackrel{\text { def }}{=} \varepsilon \frac{s^{\bullet}(s)}{s},
$$

called multiplier externality. In lack of a micro-model for multiplier effects, $\varepsilon$ is one of the main free parameters in the numerical simulations in the next section.

Utility distribution: The model distribution is assumed to depend additively on the externalities and to result from translations. That is

$$
\mu\left(\left\{u_{i}\right\} ; u\right)=\mu\left(u-\sum_{i} u_{i}\right) \mathrm{d} u .
$$

This automatically satisfies (21). In turn, (18) simplifies to

$$
\begin{aligned}
\rho^{A} & =\int_{0}^{\infty} \mu\left(u^{A}-\sum_{i} u_{i}^{A}\right) \mathrm{d} u^{A} \int_{0}^{u^{A}} \mu\left(u^{B}-\sum_{i} u_{i}^{B}\right) \mathrm{d} u^{B} \\
& =\int_{0}^{\infty} \mathrm{d} \mu\left(u^{A}\right) \int_{0}^{u^{A}+\Delta} \mathrm{d} \mu\left(u^{B}\right) \\
& =\int_{0}^{\infty} \mathrm{d} \mu\left(u^{A}\right) \mathcal{M}\left(u^{A}+\Delta\right),
\end{aligned}
$$

where

$$
\Delta \stackrel{\text { def }}{=} \sum_{i} \Delta u_{i} \stackrel{\text { def }}{=} \sum_{i} u_{i}^{A}-u_{i}^{B}
$$

is called the bias for $A$. We sometimes write $\Delta x=x^{A}-x^{B}$ for the bias of a single externality $x$. Thus, $\rho^{\bullet}=\rho^{\bullet}(\Delta)$ and it holds $\rho^{A}(\Delta)=\rho^{B}(-\Delta)$. The model specified by $(17)$ 
(27) is completely as well as numerically solvable. The explicit form of the bias is

$$
\begin{aligned}
\Delta=\Delta p-\Delta & +\Delta u_{r}+\Delta u_{m} \\
& =\left(p^{A}-p^{B}\right)+\left(\pi^{B}-\pi^{A}\right)+ \\
& +\rho^{A}\left(v_{r}^{A}-v_{r}^{B}\right)-v_{r}^{B}+\varepsilon\left(2 s^{A} / s-1\right) .
\end{aligned}
$$

Below, the shorthand $\Delta_{i} \stackrel{\text { def }}{=} \Delta u_{r}-\Delta \pi$ is frequently used.

The model above describes competition in a duopoly market in an explicit yet rather generic way by the separate construction of endogenous and exogenous externalities. For simplicity we did not employ convexity of utilities, which could be incorporated by the choice of $\mu$. The model is designed to examine competition dynamics numerically, leaving aside questions of optimality of the contracts proposed by the principals, and equilibria, cf. [26], though those are in the reach of the general model. Transaction costs are assumed to be of the same order for both goods and are thus neglected in the calculation (23) of the monetary reward cf. Section $\Pi \mathrm{II}-$ C.1. Note that in this respect the treatment of $\mathrm{A}$ and $\mathrm{B}$ as substitute goods is artificial, but can be justified for instance if the agents' budgets are limited.

Limited knowledge about the market situation entails that agents are bound to behave according to a rule of bounded rationality using partial information. The assumptions underlying the particular Ansatz are i) the price schedules $\pi^{\bullet}(s)$ are public knowledge, ii) $s$ can be estimated with good precision, as well as iii) $\rho^{\bullet}(s)$. While i) depends on the market mechanism, ii) and iii) can be justified as representing information accessible through local measurements within an agent's communication reach.

\section{EXPERIMENTS}

Two competitive situations are considered by numerical evaluation in this section. First, the phenomenon of freeriding, which is of utmost importance in the economics of information goods as detailed in Section $\mathrm{I}$ and [18], [19], is analysed in some detail. Second, we consider the competition between goods discriminated by the evolution of their respective popularities, and which are commonly called 'smash hit', respectively, 'sleeper'.

\section{A. Observables and Set-Up}

The market shares $s^{\bullet}$ and the final shares $S^{\bullet} \stackrel{\text { def }}{=} s^{\bullet}(1)$ are the primary performance figures for the comparison of the competing goods. However, the relative economic performance of the goods depends, due to the multi-level market mechanism and rewarding scheme, on the price schedule $p^{\bullet}$ and the market's dynamic evolution. This affects the agents buying and reselling the goods as well as the principals imposing the market mechanism and participating in revenues by collecting the commission determined by $\gamma^{\bullet}$.

The opportunity for the principals to extract surplus from the market depends on the (final) turnovers $t^{\bullet}(s), T^{\bullet} \stackrel{\text { def }}{=} t^{\bullet}(1)$, given by

$$
t^{\bullet}(s) \stackrel{\text { def }}{=} \int_{0}^{s} \pi^{\bullet}\left(s^{\prime}\right) \rho^{\bullet}\left(s^{\prime}\right) \mathrm{d} s^{\prime} .
$$

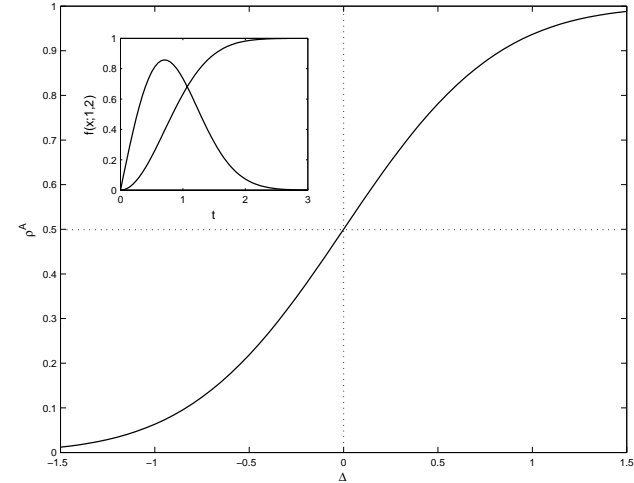

Fig. 2. Decision probability $\rho^{A}(\Delta)$ resulting from popularity distributions given by translates of the Weibull distribution $f(x ; 1,2)$. The PDF and CDF of $f(x ; 1,2)$ are shown in the inlay.

From the respective turnover, the (final) collector's revenues are derived as $c_{\gamma}^{\bullet}(s) \stackrel{\text { def }}{=}(1-\gamma) t^{\bullet}(s), C_{\gamma}^{\bullet} \stackrel{\text { def }}{=}(1-\gamma) T^{\bullet}$. They determine the economic performance of the competing good from the perspective of the principals.

As the general set-up, we specify that $\mu(\{0\} ; u)$ is given by the special Weibull distribution $f(u ; 1,2)$. The Weibull distribution is widely used in reliability and lifetime estimation. For $a=1, b=2$ it reduces to the utility distribution used in the model specification in Section III-B, namely

$$
\mu(x) \stackrel{\text { def }}{=} f(x ; 1,2)=2 x \mathrm{e}^{-x^{2}} \chi_{(0, \infty)}(x),
$$

the CDF of which is

$$
\mathcal{M}(x)=1-\mathrm{e}^{-x^{2}} \chi_{(0, \infty)}(x) .
$$

If $\mu$ is given in the form (25), formula 26) yields for $\rho^{A}(\Delta)$, $\Delta \geq 0$

$$
\rho^{A}(\Delta)=1+\frac{\sqrt{2 \pi}}{4} \Delta \mathrm{e}^{-\Delta^{2} / 2}(1-\operatorname{erf}(\Delta / \sqrt{2}))-\mathrm{e}^{-\Delta^{2} / 2} .
$$

These functions are shown in Figure 2 where it is apparent that $\rho^{A}$ is concave for $\Delta>0$, in this case, and $\rho^{A}(-\Delta)=$ $1-\rho^{A}(\Delta)$.

To reduce the parameter space of the model to finite dimensionality, we consider price schedules and popularities of the simple triangular form, with $0<s<1$,

$$
g(s ; m) \stackrel{\text { def }}{=} \begin{cases}s / m, & \text { for } 0 \leq s \leq m ; \\ (1-s) /(1-m) & \text { for } m<s \leq 1 .\end{cases}
$$

Note that this choice normalises the maximal turnover of a good, i.e., if all agents choose it, to $\int \pi^{\bullet}=0.5$. Pragmatically, $\pi^{\bullet}$ of this form is a simplification of the one in Figure 1 c). It realises a trade-off between early subscriber discount and late adopter rebate. The latter decreases and the former increases with rising $m$. In effect, the positive initial phase in the incentive schedule becomes more extended, while the negative minimum gets lower and shifted to higher $s$. 

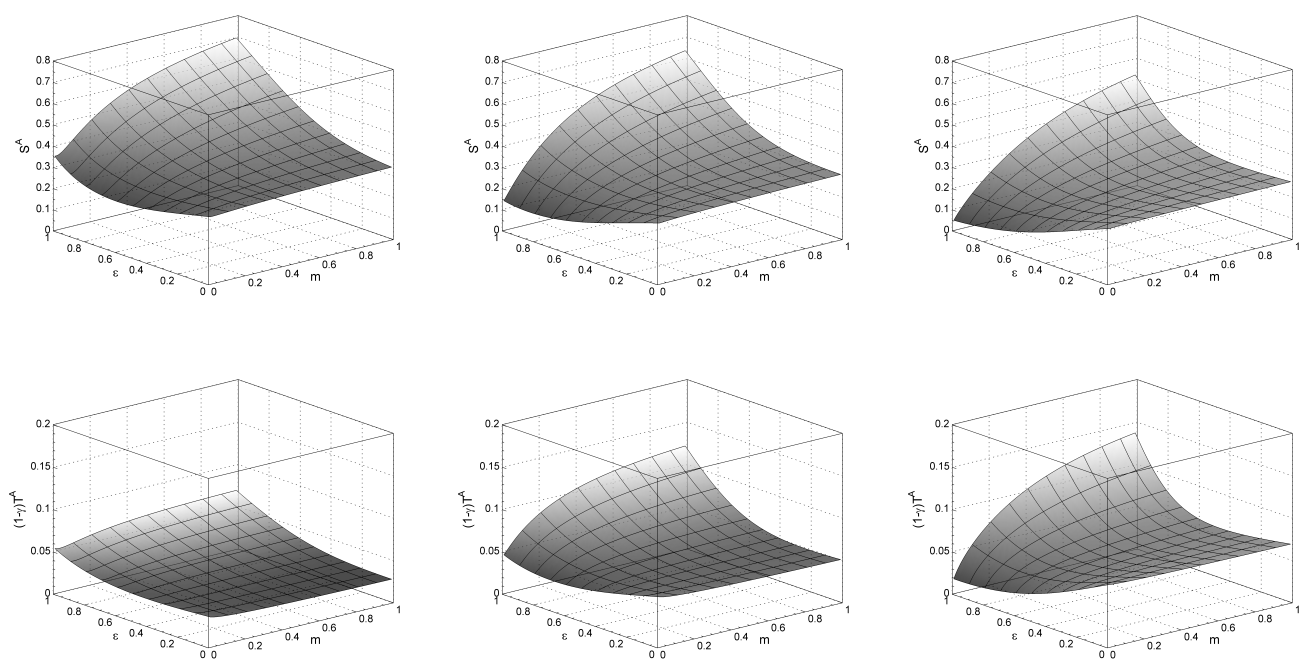

Fig. 3. Market shares (top) and collector's revenues (bottom). From left to right $\gamma=0.75,0.5,0.25$.

\section{B. Mitigation of Free-Riding}

Free riders are a fundamental problem for the marketing of information goods and to counter free-rider phenomena is the main aim behind the conception of multi-level marketing of information goods. Whether this approach can be successful is a genuine question of information economy. The presence of a free version of good $A$ renders the market similar to a pyramid scheme without a good of value. We argued in [19] that their paradoxical success hinges on distinctions between public, common, and private knowledge of the price schedule. In fact, there must be agents who know that some later adopters will have a negative incentive but expect them to buy $A$, despite having the option to choose the free version $B$. If this not the case, no dynamic equilibrium with $S^{A}>0$ can evolve. The decision rule of our model is complex enough to capture this feature and allow for nontrivial competition dynamics between an information good and its free versions.

The content distribution network systems like the Potato system [16] or Peer Impact are very similar to the peer-topeer networks commonly used by free riders [13]. By this rationale, we can compare the performance of a virtual good $A$ with a free version $B$ of it in the same market. That is, the popularities are equal $p^{A}=p^{B}$ and $B$ is free, i.e., $\pi^{B}=0$. The parameters for experimentation are $\{\gamma, m, \varepsilon\}$. We normalise the maximum of the price to 1 , as increasing the latter does not significantly improve the performance of $A$ (pertinent illustrations are omitted due to space limitations).

The first cut through the parameter space, shown in Figure 3 is taken along the $\{m, \varepsilon\}$-plane, with $\gamma=0.75,0.5,0.25$ from the left to the right column. Market shares are in the top row and collector's revenues in the bottom row. Even without a multiplier effect present, the incentive can lead to a non-negligible market share though not dominance. However significant revenues are not generated without exploiting the multiplier effect by an initial invitation to enter. For multiplier biases $\varepsilon \approx 1$ which are comparable to the price and other biases, good $A$ can reach market dominance and generate over $50 \%$ of the maximal turnover, which is $0.5(1-\gamma)$. To maximise turnovers, the price schedule must be aligned with the market growth $s^{A}$, which is generally difficult. As an effect which can be observed at low $\varepsilon$, maximisation of market shares, respectively, revenues are conflicting goals with respect to $m$ at fixed $\gamma$. On the other hand, both show a common, general characteristic. Both tend to be optimal at high $m$ and $\varepsilon$, i.e., there is a region in all graphs, in which $S^{A}$ and $T^{A}$ are both monotonic separately in $\{m, \varepsilon\}$. In this region, the incentive has a marked peak at small $s$, the positive effect for $A$ of which is amplified by a high multiplier externality.

Regarding the influence of the taxation of resellers by the collector's share $\gamma$, the latter always reduces market shares of $A$, as expected. The optimality of high $m$ becomes more marked for high taxations (low $\gamma$ ), which generally attenuate the early invitation to enter. In some cases, in particular for low $m$, the incentive for early adopters becomes negative, inverting the multiplier effect to the disadvantage of $A$, i.e., increasing $\varepsilon$ sometimes decreases $S^{A}$ and $T^{A}$.

Raising the taxation to extract a higher collector's revenue is always in conflict with obtaining dominance over free-riders in terms of market shares, as can be observed from the absolute level heights of the corresponding surfaces in the columns from left to right. From the viewpoint of mechanism design, imposing a high tax is only sensible in the presence of a significant multiplier effect, since only then can the reduced initial invitation to enter still be effective. The optimality of a late peaking price is remarkable. This penalises late market entrants who feel a strong negative pecuniary incentive, but does not affect neither shares nor revenues, since at a late stage the multiplier effect has already provided for a sufficient market share of $A$.

Some counter-arguments to imposing a late price peak have 

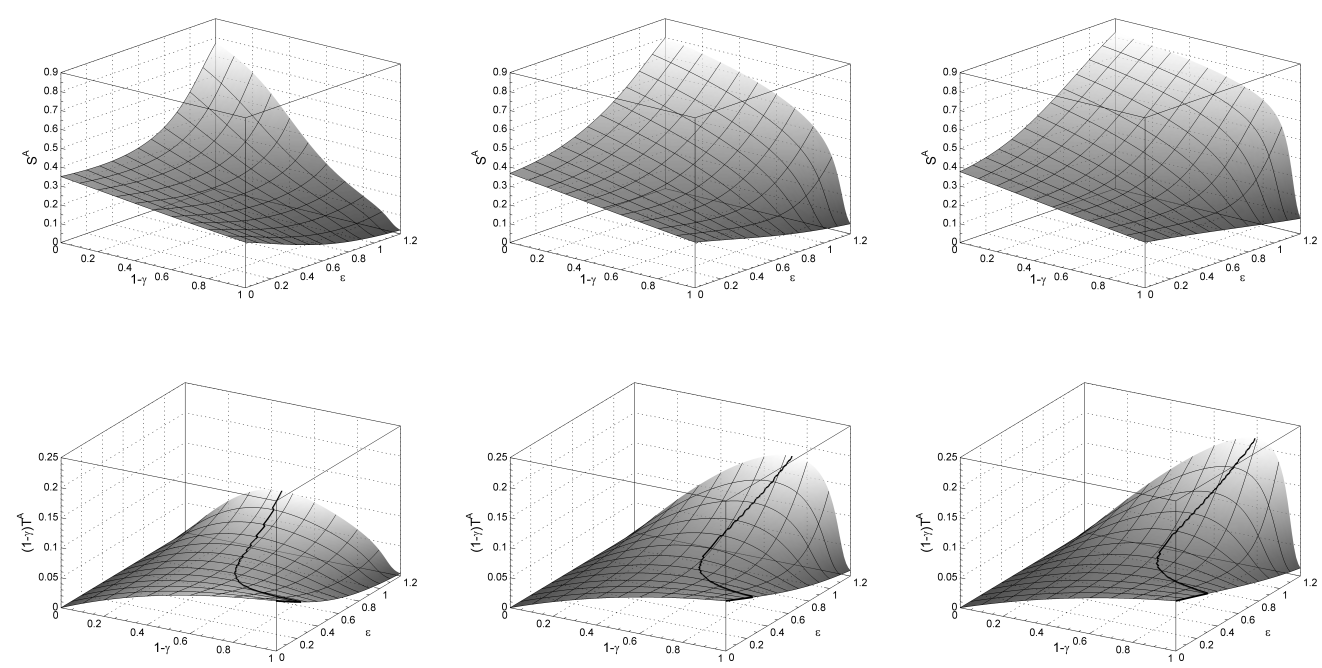

Fig. 4. Market shares (top) and collector's revenues (bottom). From left to right $m=0.1,0.5,0.9$. The line marks the maximum of $C_{\gamma}^{A}$.

been alluded to in Section II-C.2 First and foremost, the relationship of $s$ and $t$ via $n(t)$ and $n_{\infty}$ cannot be determined a priori in reality. Thus, a principal might be well-advised to offer a rebate to late subscribers in order not to loose out on a potential margin of the absolute market size. Furthermore in a dynamic setting, the price might be demand-driven, i.e., peaking with $\dot{n}(t)$, which is unlikely to happen at high saturations and late times. Finally, late adopters will find their reward expectations frustrated for high $m$, which is a strong disincentive to partake in subsequent cycles. A systematic penalty to late-comers could even be viewed as objectionable from a regulatory viewpoint.

The second cut through parameter space is taken along the $\{\gamma, \varepsilon\}$-plane, with $m$ increasing from left to right in Figure 4 . The most remarkable finding is that there exists an optimal taxation of the market at which the collector can maximise his share $C_{\gamma}^{A}=(1-\gamma) T^{A}$. The maximum is marked by the line in the graphs in the bottom row. It allows the collector to extract more than $50 \%$ of the turnover in most cases, if he is not interested in concurrently maximising the market share of $A$. The market share is always declining with $\gamma$ but depends less strongly on it for smaller $\varepsilon$. Both the $T^{A}$ and $C_{\gamma}^{A}$ are completely monotonic in $m$ for $\varepsilon \gtrsim 0.2$, reinforcing the heuristics to let the price peak as late as possible. For low $\varepsilon$ there is a region where the network market becomes ineffective for the collector. That is $\gamma=0$ is optimal there which means that resellers receive no rewards and the market would in effect return to central distribution of the good.

\section{Competition Between Smash Hit and Sleeper}

Scenarios for the competition of two goods are manifold within our model and a comprehensive treatment is beyond the scope of the present paper. In general, the goods $A$ and $B$ can differentiate in price schedule and popularity in the generic model of Section III-B Here, as a familiar example, good $A$ is assumed to have a popularity function peaking later than that of $B$, i.e., $A$ would commonly be termed a 'sleeper' while $B$ can be considered a 'smash hit'. Concretely we let $p^{A}$ and $p^{B}$ be functions of triangular shape (33), with peaks at $2 / 3$, respectively, $1 / 3$. For further simplification we consider the case $\gamma^{\bullet}=0$, leaving us with $m^{A}, m^{B}$ as the only discriminating factors in the competition. This oversimplified situation shall give a first impression of the qualitative traits of competition in network markets.

As observables beside the shares $S^{A}$ and turnovers $T^{A}$ of good $A$ we consider the combined turnover $T=T^{A}+T^{B}$ in the top, middle, and bottom row of Figure 5 respectively. The combined turnover carries unique economic information. Since we can expect the competing goods to introduce some friction into the market, $T$ will also be decreased from the maximum that is achievable given the price schedules of $A$ and $B$. In the generic dynamics of competition expressed by formula (17) we have $\rho^{A}=1-\rho^{B}$, from which follows

$$
T=T_{0}^{A}+T_{0}^{B}-\int_{0}^{1} \rho^{A} \pi^{B}+\rho^{B} \pi^{A} \mathrm{~d} s
$$

by an easy calculation. Here $T_{0}^{\bullet} \stackrel{\text { def }}{=} \int_{0}^{1} \pi^{\bullet}$ is the maximum turnover without competition. This equation expresses the mentioned friction and has a straightforward interpretation. The effect occurs when one good has a high utility and the other a high price. If the price were the only externality, this would not happen, but in a network market the incentive and other utilities which are not synchronised with the price schedule can cause non-negligible friction.

As for the shares (top row of Figure 5], the first observation is that they are decreasing in $\varepsilon$ for $\varepsilon \lesssim 0.8$. Regardless of discrimination by price and incentive schedules, $B$ profits more from multiplier effects in this region, due to higher popularity at early times. A's share generally develops a maximum for given $m^{B}$ in a region below the diagonal 

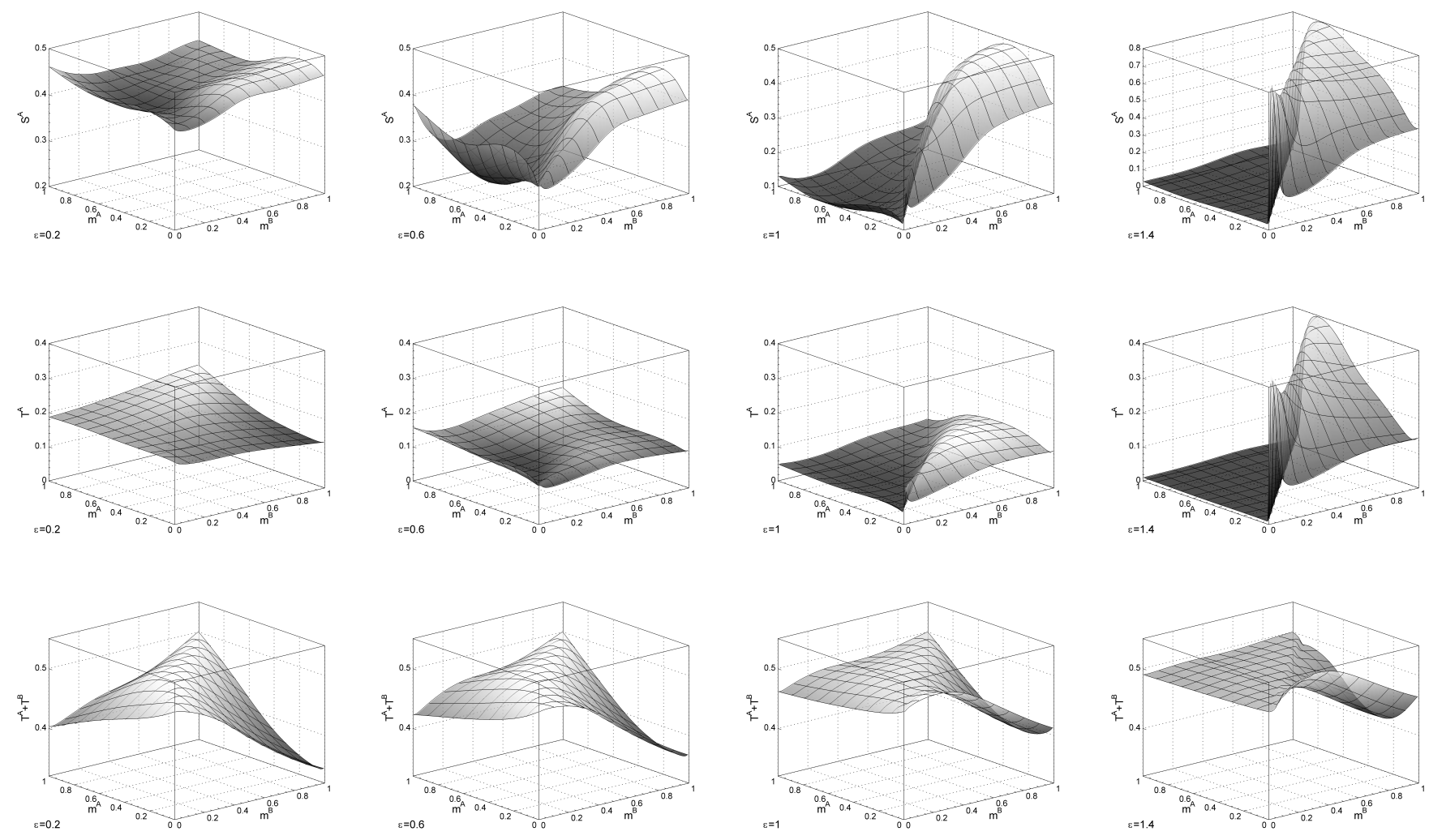

Fig. 5. Competition between smash hit and sleeper. Top row: Market shares of $A$. Middle row: Turnovers of $A$. Bottom row: Combined turnovers

$m^{A}=m^{B}$. If the price of $A$ peaks earlier than $B$ 's, then the expected incentive will be higher for $A$ during a short initial period. This is the basic effect from incentives that $A$ 's principal can use to his advantage. The opposite effect lets $S^{A}$ attain a local minimum above the diagonal. During the next phase in the market evolution, $u_{i}^{B}$ is larger than $u_{i}^{A}$, producing a counter-effect which significantly decreases $A$ 's share for too low $m^{A}$ and becoming more expressed as it is amplified by a high multiplier factor. The transition occurring at the local maximum of $S^{A}$ becomes sharper with rising $\varepsilon$.

The situation is even worse for the turnovers generated from A. $T^{A}$ develops no significant maximum below $\varepsilon \approx 0.8$. $A$ 's principal cannot maximise turnovers in this situation. Moreover, as can be observed in the leftmost and second to left column, $T^{A}$ decreases precisely in the region where $A$ 's market shares are maximised for $\varepsilon \lesssim 0.6$. That is that an early advantage of $A$ is not sustained long enough, due to lack of strength of the network effect, to allow for generation of turnovers when $\pi^{A}$ peaks. In this case $A$ conquers market shares only by price dumping. The mentioned price dumping also introduces friction in the market, reducing $T$ in the region of parameters just described.

In conclusion, to counter the slow startup effect due to later popularity utilising an appropriate price schedule, $A$ 's principal cannot simply employ a long-lasting discount at early times, but needs to carefully align his rewarding schedule with that of $B$. This is difficult if $\pi^{B}$ is not known. To optimise the price function of the sleeper so as to obtain significant market shares and turnovers, is yet more difficult, and generally only possible if significant network externalities can be exploited.

\section{CONClusion}

We have exhibited in Section [II the general macroscopic mechanism of network and multi-level markets in a continuous, dynamic setting. To the best of our knowledge, no comparable model for the monetary flux in these markets exists. A remarkable characteristic of the model is the simplicity and coherence by which it is constructed from first principles. This enables the rigorous derivation of important features such as the balance condition (4) and the inversion formula (6), in turn enabling mechanism design by dynamic forward pricing. Section [I-C exhibits some of the rich, realistic features of the model. The expression (11) for revenues from down-line payments derived in Section II-B is a textbook-example of a Markov process. The limit case of infinite levels of rewards is tractable and yields the nontrivial result that properly designed multi-level markets yield a benefit to all buyers.

The basic assumption of market homogeneity, cf. Section IIC.5. can be relaxed in refined models. One direction explored in the model for competition of Section [II] is the inclusion of network effects, externalities to which the monetary flux model is neutral due to its scale-freeness, cf. Section III-C.3. Experiments in the duopoly competition framework, cf. Section IV-B 
answer the central question of the Introduction affirmatively. Network marketing of information goods is a viable approach to mitigate free-riding. While in the case of free-riding, a lot is known about the competing good, e.g., its price is zero and the popularity approximately equals that of the original, the dynamic evolution of the relative demands for original and pirated version is not easy to determine. Therefore, finding the optimal price and rewarding schedule is difficult even in this simple case. For the design of optimal market mechanisms further factors should be taken into account as discussed in Sections II-C.2, IV-B In particular, the penalty to late adopters remains a critical issue in strategic planning of sustainable markets designed to operate for a long time and with many goods. An approach suggesting itself to improve the performance of the original is positive discrimination by factors like added quality or non-monetary rewards.

The competitive situation between two different goods can become rather complicated as we have seen in Section IVC. Without very detailed, and even a priori, information on the superior good's - the smash hit's - demand curve, the sleeper's principal has little chance to gain a significant market share. Thus, the price schedule is not a very effective tool in the general competition of goods in the same network marketing structure. We conclude that network markets are open and non-discriminatory. They can offer superior market mechanisms in particular in the presence of network effects, and in cases when the competitor's characteristics are rather special and known beforehand, like for pirated versions. Pragmatically another difficult task for mechanism design is to ensure equal opportunities for resellers in the market, i.e., to underpin the assumption of homogeneity.

We close the discussion with some directions for further work. Properties of real network markets should, however, be justified on the micro-economical level. To complement theoretical approaches by simulation studies of the dynamics of MLIM markets, using the methods of agent-based computational economics [27], such as [13], is desirable. They would allow to study the influence of particularities (topologies) of the communication network underlying the market, and address the issue of inhomogeneities. In particular, a better justified microscopic model for the multiplier effect is wanting.

A proper treatment of network and multi-level markets and the competition of goods therein from the viewpoint of theoretical economics should also answer questions of optimality, equilibria, and their stability. The proper theoretical framework for this is the principal-agent model [26]. In particular in the free-rider situation it could be used to describe the effect of the incentive from multi-level rewards on the moral hazard incurred by the agents.

\section{ACKNOWLEDGEMENT}

Thanks go to Nicolai Kuntze for numerous discussions.

\section{REFERENCES}

[1] C. Shapiro and H. Varian, Information Rules: A Strategic Guide to the Network Economy. Harvard Business School Press, 1999.
[2] M. Stegman, "Information goods and advertising: An economic model of the internet." in National Bureau of Economic Research Universities Research Conference 'Economics of the Information Economy'. 7 and 8 May 2004, Royal Sonesta Hotel, Cambridge, MA., 2004. [Online]. Available: http://www.nber.org/ confer/2004/URCs04/stegeman.pdf

[3] "Digital millennium copyright act," Public Law 105-304, 105th Congress, 112 Stat. 2860, October 28 1998. [Online]. Available: http://en.wikisource.org/wiki/Digital_Millennium_Copyright_Act

[4] "WIPO copyright treaty and agreed statements concerning the WIPO copyright treaty," Adopted in Geneva on 20 December, 1996. [Online]. Available: http://www.wipo.int/edocs/trtdocs/en/wo/wo033en.htm

[5] "On the harmonisation of certain aspects of copyright and related rights in the information society," Directive 2001/29/EC of the European Parliament and of the Council, May 222001. [Online]. Available: http://eur-lex.europa.eu/LexUriServ/LexUriServ.do? uri=CELEX:32001L0029:EN:HTML

[6] E. Becker, W. Buhse, D. Günnewig, and N. Rump, Eds., Digital Rights Management - Technological, Economic, Legal and Political Aspects, ser. Lecture Notes in Computer Science. Berlin, Heidelberg: SpringerVerlag, 2003, vol. 2770.

[7] P. Drahos and I. Maher, Eds., Information Economics and Policy. Special Issue. Elsevier, January 2004, vol. 16.

[8] D. K. Mulligan, J. Han, and A. J. Burstein, "How DRM-based content delivery systems disrupt expectations of "personal use'," in Proceedings of the ACM workshop on Digital Rights Management, 2003, pp. 77-89.

[9] P. Aichroth and J. Hasselbach, "Incentive management for virtual goods: About copyright and creative production in the digital domain," in International Workshop on Virtual Goods, 27-29 May 2003. Technical University Ilmenau, Germany, 2003, pp. 70-81. [Online]. Available: http://virtualgoods.tu-ilmenau.de/2003/incentive_management.pdf

[10] J. Chaffin, K. A. in New York, A. Edgecliffe-Johnson, and D. Ibison, "Apple sparks battle over copyright," FT.com, February 7 2007. [Online]. Available: http://www.ft.com/cms/s/ 90c03168-b6ea-11db-8bc2-0000779e2340.html

[11] S. Jobs, "Thoughts on music," Open Letter, February 6 2007. [Online]. Available: http://www.apple.com/hotnews/thoughtsonmusic/

[12] S. M. Fulton III, "Jupiter analyst: Interoperable drm won't solve music industry dilemma," BetaNews, February 16, 5:58 PM 2007. [Online]. Available: http://www.betanews.com/article/Jupiter_Analyst_Interoperable_ DRM_Wont_Solve_Music_Industry_Dilemma/1171664985/1

[13] K. G. Zerfiridis and H. D. Karatza, "File distribution using a peer-topeer network - a simulation study," Journal of Systems and Software, vol. 73, pp. 31-44, 2004.

[14] "Peer Impact," Web Site, visited June 9 2007. [Online]. Available: http://www.peerimpact.com/

[15] R. Grimm and J. Nützel, "Security and business models for virtual goods," in Proceedings of the 2002 ACM Multimedia Security Workshop, 2002, pp. 75-79.

[16] J. Nützel and R. Grimm, "Potato system and signed media format — an alternative approach to online music business," in Proceedings of the 3rd International Conference on Web Delivering of Music (WEDELMUSIC 2003). IEEE Press, 2003, pp. 23-26.

[17] H.-W. Micklitz, B. Monazzahian, and C. Rößler, "Door-to-door selling — pyramid selling — multilevel marketing." Study commissioned by the European Commission. Contract No. A0/7050/98/000156, 1999. [Online]. Available: http://europa.eu.int/comm/dgs/health_consumer/ library/surveys/sur10_en.html

[18] A. U. Schmidt, "Multi-level markets and incentives for information goods," Information Economics and Policy, vol. 18, no. 2, pp. 125-138, 2006. [Online]. Available: http://andreas.schmidt.novalyst.de/ docs/Incentive_Systems_IEP_revised.pdf

[19] - "Multi-level markets for virtual goods," in Axmedis 2005, Volume for Workshops, Industrial, and Application Sessions, ser. Atti, P. Nesi, K. Ng, and J. Delgado, Eds., vol. 22. Firenze University Press, 2005 , pp. 134-141. [Online]. Available: http://arXiv.org/abs/cs.GT/0409028

[20] B.-L. Lim, M. Choi, and M.-C. Park, "The late take-off phenomenon in the diffusion of telecommunication services: network effect and the critical mass," Information Economics and Policy, vol. 15, no. 4, pp. 537-557, 122003.

[21] N. Economides, "The economics of networks," International Journal of Industrial Organization, vol. 14, pp. 673-699, 1996.

[22] — "Network externalities, complementarities, and invitations to enter," European Journal of Political Economy, vol. 12, pp. 211-233, 1996. 
[23] G. M. P. Swann, "The functional form of network effects," Information Economics and Policy, vol. 14, no. 3, pp. 417-429, 92002.

[24] M. J. Salganik, P. S. Dodds, and D. J. Watts, "Experimental study of inequality and unpredictability in an artificial cultural market," Science, vol. 311, no. 5762, pp. 854-856, 2 2006. [Online]. Available: http://www.sciencemag.org/cgi/content/abstract/311/5762/854

[25] S. M. Maurer and B. A. Huberman, "Competitive dynamics of web sites," Journal of Economic Dynamics and Control, vol. 27, pp. 21952206, 2003

[26] J.-J. Laffont and D. Martimort, The Theory of Incentives. Princeton and Oxford: Princeton University Press, 2002.

[27] L. Tesfatsion, "Introduction to the special issue on agent-based computational economics," Journal of Economic Dynamics and Control, vol. 25, pp. 281-293, 2001. 